\title{
Ion feedback suppression using inclined MCP holes in a "Single-MCP+Micromegas+Pads" Detector
}

\author{
J.Va'vra, SLAC, Stanford, CA 94305, USA* \\ T. Sumiyoshi, Tokyo Metropolitan University, Tokyo, Japan ${ }^{* *}$
}

\begin{abstract}
We show that the ion backflow to photocathode can be completely suppressed if one uses inclined MCP holes in a presence of magnetic field. The inclined hole angles are chosen to be aligned with the Lorentz electron angle allowing the electron transmission and amplification, while positive ions, traveling along a different angle, are caught on the MCP hole walls. The detector under investigation is a new gaseous detector structure based on a tandem of two parts, a single MCP (Microchannel) plate, a Micromegas with pad readout. We are aiming to develop a gaseous photon detector with the Bialkali photocathode, however, one could use some ideas in the paper for other types of detectors.
\end{abstract}

Keywords: Photodetectors; Ion backflow suppression; Cherenkov detectors; RICH.

\section{INTRODUCTION}

This presentation represents a continuation of the R\&D effort to develop a permanently sealed gaseous photo-detector with the Bialkali photocathode. After several attempts, we have gravitated towards a geometry based on the single MCP with Micromegas and pad readout [1]. The operation seems to be very good relative to any other photo-detector we have worked with in past, and that includes, for example, the CRID [2] or quadruple-GEM [3] detectors. The reason for choosing the MCP rather than the GEM structure is twofold: (a) it is made with much higher purity process and therefore a long-term poisoning of the Bialkali photocathode is less likely, and (b) it allows the angled holes and therefore it allows the new ion backflow suppression ideas described in this paper.

It would appear on the surface that choosing a gaseous device is a step backward. However, there are several attractive points of the gaseous detectors compared to the vacuum-based devices:

(a) First, the micro-pattern gas detectors have much better aging rate than their predecessors: the wire chambers. They can also handle much higher rates, as the COMPASS experiment [4] proved.

(b) One can use rather large MCP hole of $50 \mu \mathrm{m}$ diameter even at $15 \mathrm{kG}$, thus making the MCP to be a standard and therefore cheaper compared to the vacuum-based MCP-PMTs, which require a hole diameter of $6-10 \mu \mathrm{m}$ to run at $15 \mathrm{kG}$ without a substantial gain loss. One could also worry that smaller hole diameter means more difficulty in scrubbing the device to reach excellent vacuum. Poor vacuum means higher cathode aging rate as the residual gas produces ions by electron bombardment. The resulting aging effects must not be underestimated. For example, if the present BaBar DIRC would be built with the early MCP-PMTs, it would die after two years. Finally, our experience indicates so far that the MCP-PMTs with smaller hole diameters are more noisy.

** Work supported by the Department of Energy, contract DEAC02-76SF00515.

* Work supported in part by a Grand-in-Aid for Scientific Research from JSPS under Grant No. 14340083 and the Japan/U.S. cooperation program in the field of high energy physics 
(c) Larger MCP hole diameter also means a high electron collection efficiency $>98 \%$. This has been demonstrated experimentally with the GEM structure where the hole diameter is typically $\sim 50 \mu \mathrm{m}$. In contrast, the vacuum-based MCP-PMTs have the collection efficiency typically less than $70 \%$.

(d) In principle, another advantage of operation in the gas is a reduction of ion energy, which could reduce the damage of photocathode by the ion bombardment. However, there are many more ions compared to the vacuum devices, and these ions participate in the secondary cathode processes when they reach the Bialkali photocathode, causing the secondary photoelectron production. This effect causes a faster than exponential gain increase, and this reduces a maximum achievable gas gain. If such behavior would be allowed, it would be difficult to bring a large system into a stable operation. There are various attempts to overcome this problem, usually achieved by clever but complicated tricks with the electrostatic arrangements. We have invented a simple method to block the ion flow to the cathode. The method consists of using the inclined MCP holes, which are aligned with the electron Lorentz angle in the gas at a given magnetic field.

One should note that the trapped ions on the MCP walls could also generate the secondary effects. If this happens, there would not be much advantage from the proposed geometry. However, we assume that it is less likely compared to the photocathode surface covered by a very sensitive material. Furthermore, the diffusion of electron cloud during the MCP amplification is large, and therefore any build up of ions on the MCP walls, which could trigger the Malter effect type of electron emission, will have a better chance to neutralize with electrons. We would like to also move toward more conducting glasses in future, for example soda glass [5]. Finally, the most important argument is that the MCP gain is low, i.e., less than 10-20. Most of the gain will be provided by the Micromegas part $\left(\sim 10^{4}\right)$.

To achieve high QE in the gaseous photo-detector, the photoelectron backscattering on the gas molecules towards to the photocathode, where they can be captured, has to be small. In this sense, the helium or argonbased gases with a large percentage of the noble gas component are not ideal. Instead, molecules vibrating with more degrees of freedom are better, such as pure hydrocarbons gases $\left(\mathrm{CH}_{4}, \mathrm{C}_{2} \mathrm{H}_{6}\right.$ or $\left.\mathrm{iC}_{4} \mathrm{H}_{10}\right)$, or a pure $\mathrm{CF}_{4}$ gas [6]. Although our initial testing was done with a $90 \% \mathrm{Ar}+10 \% \mathrm{CH}_{4}$ gas, we plan to switch to pure $\mathrm{CH}_{4}$ gas.

Timing with the gaseous detectors is likely to be worse compared to the vacuum devices. However, Giomataris has achieved a timing resolution of $\sigma \sim 300 \mathrm{ps}$ with a single Micromegas operating with single photoelectrons. This was done with a simple leading edge discriminator, and one would hope that this would get better with a CFD discriminator. Although the MCP gain mechanism itself, and especially a photoelectron entry into its holes, will undoubtedly create similar timing tails observed typically with the vacuum-based MCP-PMTs, nevertheless, we are eager to measure it.

This paper shows for the first time, to our knowledge, that it is possible to either eliminate the ion feedback entirely, or, reduce it to a very small level by aligning the MCP holes with the electron Lorentz angle. The ions follow a different path and are caught on the MCP holes. In a geometry, where one can arrange the MCP electric field to have a large angle with the magnetic field, one can indeed achieve this, and this paper provides a proof. For example, such geometry could be easily achieved in the Focusing DIRC by suitably rotating the focal plane. 


\section{CALCULATION OF THE LORENTZ ANGLE}

Fig. 1 shows the principle of the proposed detector. Typically, the operating parameter space of this type of detector would be [1]: $\mathrm{E}_{\mathrm{MCP} \text {-entrance }} \sim 350 \mathrm{~V} / \mathrm{cm}, \mathrm{E}_{\mathrm{MCP} \text {-exit }} \sim 1.25 \mathrm{kV} / \mathrm{cm}, \mathrm{E}_{\mathrm{MCP}}$ up to $10 \mathrm{kV} / \mathrm{cm}$, and $\mathrm{E}_{\text {Micromegas }}$ up to $50 \mathrm{kV} / \mathrm{cm}$, corresponding to an average gain of a $2-3 \times 10^{5}$.

For the presented idea to work, it is crucial to assume that the electric field in the MCP with the inclined holes is perpendicular to the faceplates ${ }^{1}$, i.e., not aligned with the MCP hole direction. The next step is to calculate the Lorentz angle. To do this calculation, we used a computer program Magboltz, version 7.1 [7]. Fig. $2 \mathrm{a}$ shows a calculation of the Lorentz angle as a function of electric field gradient in $90 \% \mathrm{Ar}+10 \% \mathrm{CH}_{4}$, $\mathrm{CH}_{4}$ and $\mathrm{CF}_{4}$ gases. The electric and magnetic field are perpendicular to each other and magnetic field is $\mathrm{B}=$ $15 \mathrm{kG}$. The Lorentz angle is $\sim 6.5^{\circ}$ at $\mathrm{E}_{\mathrm{MCP}} \sim 10 \mathrm{kV} / \mathrm{cm}$ in $90 \% \mathrm{Ar}+10 \% \mathrm{CH}_{4}$. Assuming the MCP thickness of $1 \mathrm{~mm}$ and the MCP hole diameter of $25 \mu \mathrm{m}$, one can achieve a complete opacity for the ions with such an angle. Fig. $2 \mathrm{~b}$ shows a similar calculation as a function of the $\mathrm{Ar} / \mathrm{CH}_{4}$ gas mix ratio at $\mathrm{E}_{\mathrm{MCP}}=9 \mathrm{kV} / \mathrm{cm}$. One can see that the Lorentz angle is not very sensitive to the exact mix ratio.

The calculation also indicates relatively large electron diffusion within the $\mathrm{MCP}$ holes: $\sigma_{\text {long_(along_E) }}$ $\sim 106 \mu \mathrm{m}^{2} / \mathrm{ns}$ and $\sigma_{\text {transv_(along_B }} \sim 245 \mu \mathrm{m}^{2} / \mathrm{ns}$ in $90 \% \mathrm{Ar}+10 \% \mathrm{CH}_{4}$ for $\mathrm{E}_{\mathrm{MCP}}=9 \mathrm{kV} / \mathrm{cm}$ and B $=15 \mathrm{kG}$, with corresponding calculated average drift velocity is $\mathrm{v}_{\text {along_E }} \sim 36.75 \mu \mathrm{m} / \mathrm{ns}$ and $\mathrm{v}_{\text {along_B }} \sim 4.21 \mu \mathrm{m} / \mathrm{ns}$. This means that some electrons will be lost on the walls, or more likely participate in the electron-ion recombination along the MCP walls.

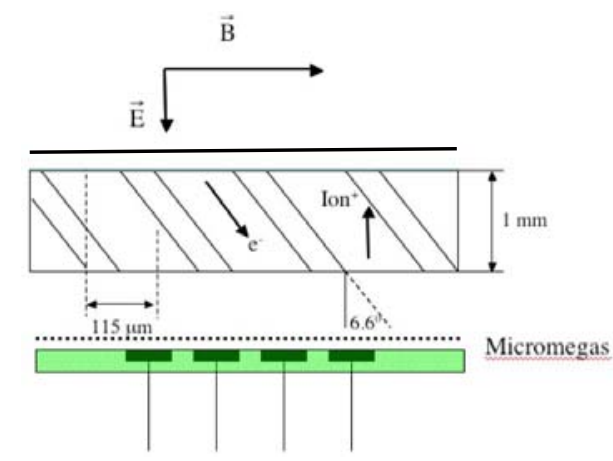

Fig. 1. Principle of a detector "single MCP+Micromegas+pads" with the inclined MCP holes for a geometry where E and B are perpendicular to each other. The MCP holes are chosen to be the same as the electron Lorentz angle. The backflow ions, following a different angle, are trapped by hole walls.

\footnotetext{
1 This assumption was confirmed by Hamamatsu and Burle.
} 
(a)

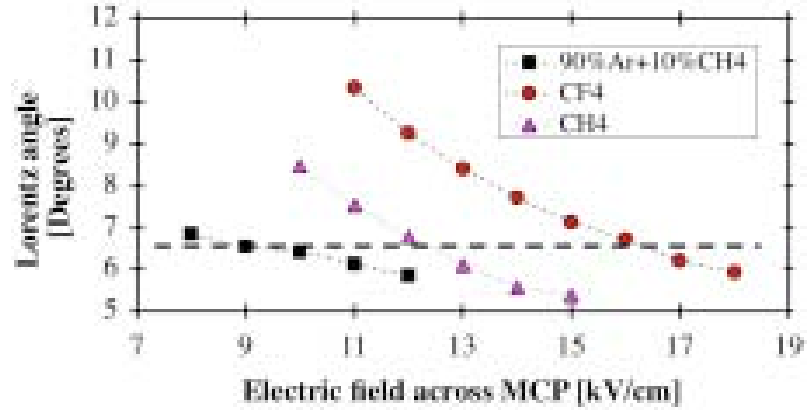

(b)

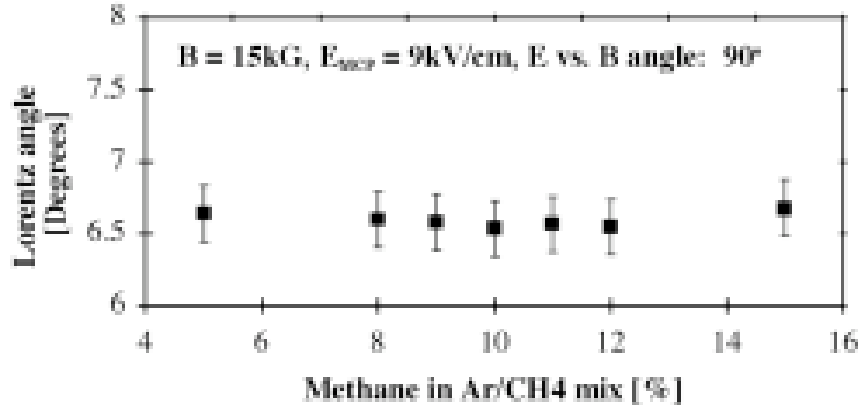

Fig. 2. (a) Calculation of the Lorentz angle as a function of electric field gradient in $90 \% \mathrm{Ar}+10 \% \mathrm{CH}_{4}$, $\mathrm{CH}_{4}$ and $\mathrm{CF}_{4}$ gases. The electric and magnetic field are perpendicular to each other and magnetic field is $\mathrm{B}=15 \mathrm{kG}$. The line indicates a $6.5^{\circ}$ angle of our MCP holes. (b) The same calculation as a function of $\mathrm{Ar} / \mathrm{CH} 4$ gas mix ratio for the electric field gradient $\mathrm{E}_{\mathrm{MCP}}=9 \mathrm{kV} / \mathrm{cm}$.

\section{EXPERIMENTAL SETUP AND MEASUREMENTS}

Ref. 1 showed already that the ion backflow in a MCP with the perpendicular holes could be substantial. In this paper we have repeated the measurement with higher accuracy. Fig. 3 shows the experimental setup. These measurements were with the magnetic field off. The photocurrent was generated with the Mercury UV lamp and a dense s.s. mesh defining the cathode in this detector. Fig. 4 shows that the ion feedback is substantial, close to $\sim 10 \%$, when varying the gain with the MCP voltage, and close to $\sim 3 \%$, when varying the gain with the Micromegas voltage. Both numbers are significant for any practical application.

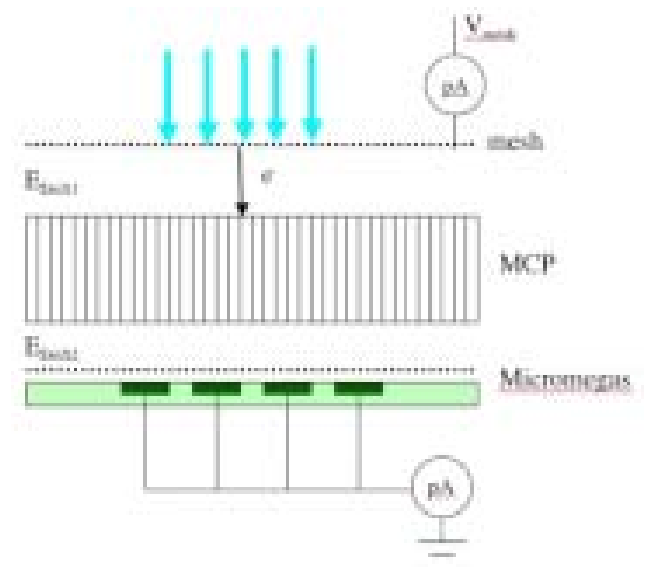

Fig. 3. Experimental setup with a "single MCP+Micromegas+pads" detector to measure the ion backflow to cathode. The MCP has perpendicular holes (angle $0^{\circ}$ ) and $\mathrm{B}=0 \mathrm{kG}$. 
(a)

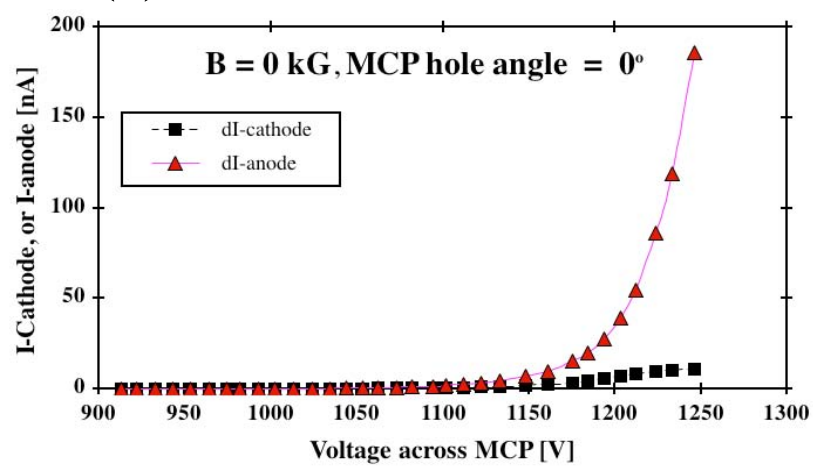

(b)

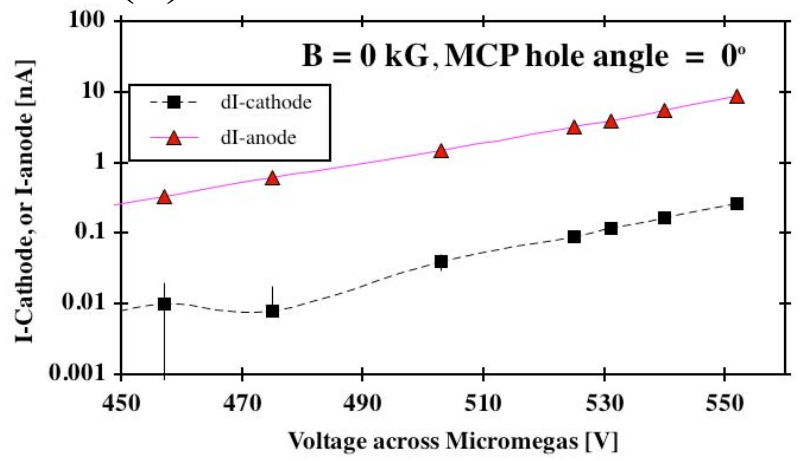

Fig. 4. The ion backflow rate: (a) As a function of the MCP voltage in the experimental setup of Fig. 3 (MCP hole angle $0^{\circ}$ and $\mathrm{B}=0 \mathrm{kG}$ ). The cathode current is about $10 \%$ of the anode current at $\mathrm{V}_{\mathrm{MCP}}=1200 \mathrm{~V}\left(\mathrm{E}_{\mathrm{MCP}}=12 \mathrm{kV} / \mathrm{cm}\right)$ and in $90 \% \mathrm{Ar}+10 \% \mathrm{CH}_{4}$ gas. (b) As a function of the Micromegas voltage (MCP hole angle $0^{\circ}, \mathrm{B}=0 \mathrm{kG}$, and $90 \% \mathrm{Ar}+10 \% \mathrm{CH}_{4}$ gas). The cathode current is about $3 \%$ of the anode current for this particular choice of parameters.

(a)

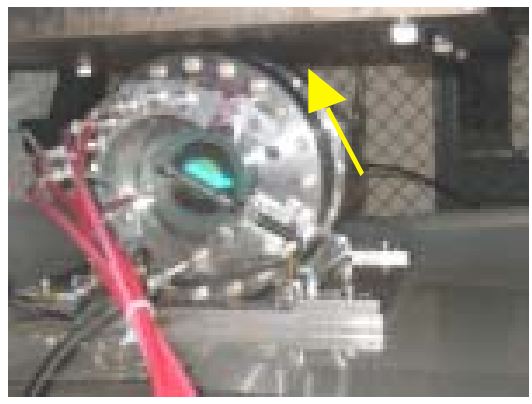

(b)

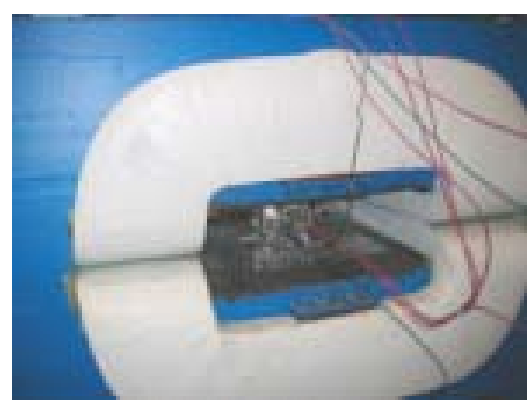

Fig. 7. Experimental setup in a $15 \mathrm{kG}$ dipole magnet. It uses a Mercury UV lamp. The detector vessel can rotate to find an optimum angle at which the electron transmission is at maximum. The arrow indicates the direction of rotation. 
At this point we switched to a MCP with inclined holes. Fig. 5 shows the experimental setup to measure the ion backflow in the magnetic field. The magnetic field is chosen to be perpendicular to electric field. Fig. 6 shows a cut through our MCP indicating the inclined hole angle if $6.5^{\circ}$. Fig. 7 shows the actual experimental vessel placed in the dipole, which was capable of delivering up to $15 \mathrm{kG}$.

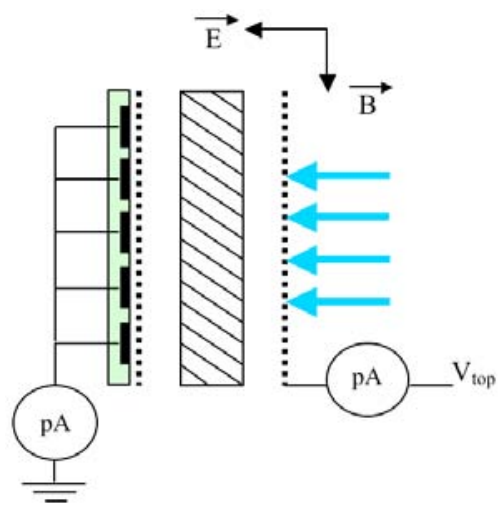

Fig. 5. Experimental setup a "single MCP+Micromegas with pads" detector to measure the ion backflow to cathode. The MCP has inclined holes (angle $6.5^{\circ}$ ) and $\mathrm{B}=15 \mathrm{kG}$.

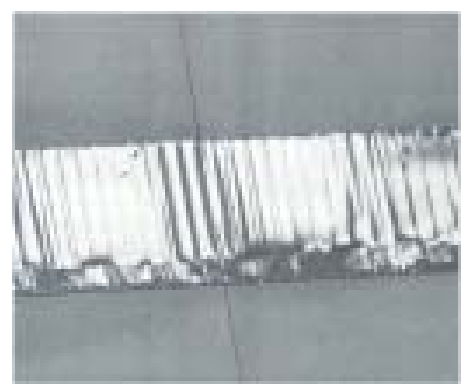

Fig. 6. Cut through the Hamamatsu MCP with the inclined holes, which is used in the tests of this paper. The MCP hole angle is $6.5^{\circ}$.

To achieve an optimum electron transmission through the angled hole, i.e., to align the MCP hole with the electron Lorentz angle, it is necessary to rotate the MCP around its axis. This is achieved by rotating the entire detector vessel. Fig. 8 shows a result of such tuning as a function of the MCP azimuth angle. At this angle, the anode current is at maximum, and the ion backflow current is very small, and, in fact, consistent with a pico-ammeter noise of $\sim 5 \mathrm{pA}$. The noise was somewhat larger than a usual $<1 \mathrm{pA}$ level because the Kiethley pico-ammeters are very strongly affected by the magnetic field and it was necessary to place them far away from the magnet, and therefore far from the detector.

Once the optimum azimuth was chosen, we proceeded with the measurement of the ion backflow. Fig. 9 shows the ion backflow measurement as a function of Micromegas voltage for several choices of MCP electric gradient. In each case, the ion backflow current is negligible relative to anode current, and consistent with the pico-ammeter noise of $\sim 5 \mathrm{pA}$.

Fig. 10 shows the relative gain as a function of the voltage across Micromegas for several choices of electric gradient across MCP. One can see a usual exponential behavior. If one does the same gain curve as a 
function of MCP voltage, it is convoluted with the electron acceptance in the holes due to changing Lorentz angle as a function of $\mathrm{E}_{\mathrm{MCP}}$. Resulting gain curve is still exponential, but less steep.

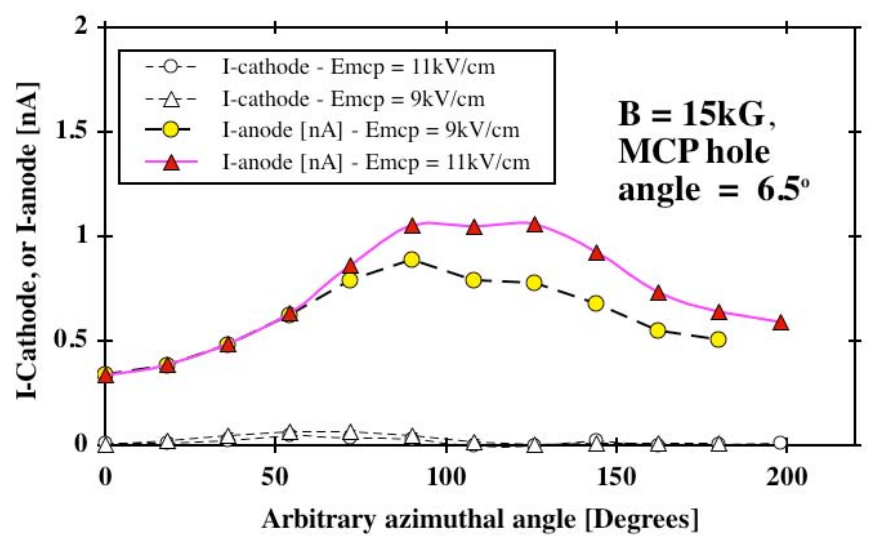

Fig. 8. Experimental tuning of the MCP azimuth angle to achieve the best electron transmission and the optimum alignment. At this angle, the ion backflow current is very small and consistent with the pico-ammeter noise of $\sim 5 \mathrm{pA}$. The gas is $90 \% \mathrm{Ar}+10 \% \mathrm{CH}_{4}$.

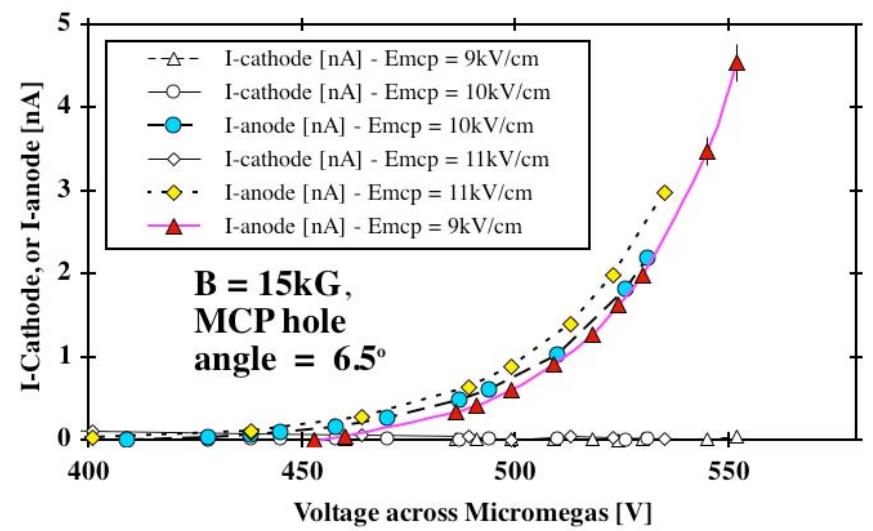

Fig. 9. The ion backflow measurement as a function of Micromegas voltage for several choices of MCP electric gradient. In each case, the ion backflow current is negligible, i.e., consistent with the picoammeter noise. The gas is $90 \% \mathrm{Ar}+10 \% \mathrm{CH}_{4}$.

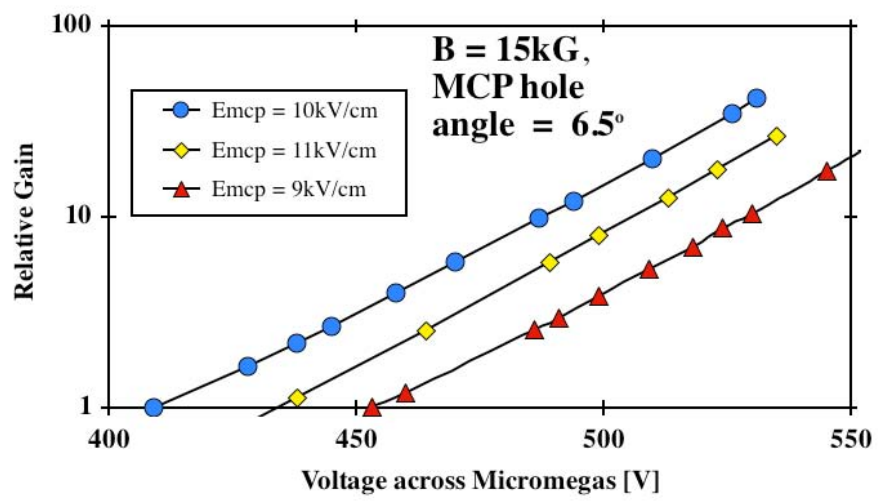


Fig. 10. Relative gain as a function of voltage across the Micromegas for several choices of electric gradient across the $\mathrm{MCP}$ in $90 \% \mathrm{Ar}+10 \% \mathrm{CH}_{4}$ gas.

It is clear that the detector with the inclined MCP holes has no gain for $\mathrm{B}=0 \mathrm{kG}$, and it cannot be tested easily in a lab without a magnet. That represents a certain operational difficulty in early R\&D stages. However, during a real experiment, typical conditions are always with the magnetic field on.

\section{BIALKALI GASEOUS PHOTO-DETECTOR BY HAMAMASTU}

To start the initial study, Hamamatsu has constructed a double-Micromegas detector described on Fig. 11. It has two-mesh structure, Bialkali photocathode, all enclosed in a standard glass envelope. The aim is to overcome several difficulties if one wants to connect a gaseous detector to the Bialkali photocathode, namely:

(a) The gas and materials involved have to be pure and chemically inert to ensure the photocathode longevity, both during the photocathode production and during a normal operation. One must avoid especially low ionization potential impurities, which would deliver a charge to the cathode (because of the charge exchange process), where they could either plate on the surface or interact chemically with the photocathode. In other words, ions must be made of species of the main gas filling, and return back to the original gas molecule after the charge recombination. With our proposed detector, a majority of ions will be recombined on the Micromegas mesh, and the remaining ions on the MCP inclined holes. The diffusion during the electron amplification in the MCP holes is very large, which means that electrons are sweeping the MCP holes and making ions to recombine.

(b) The photoelectron backscattering on the gas molecules towards to the photocathode, where they can be captured, has to be small. In this sense, the helium or argon-based gases with a large percentage of the noble gas component are not ideal. Instead, molecules vibrating with more degrees of freedom are better, such as pure hydrocarbons gases $\left(\mathrm{CH}_{4}, \mathrm{C}_{2} \mathrm{H}_{6}\right.$ or $\left.\mathrm{iC}_{4} \mathrm{H}_{10}\right)$, or a pure $\mathrm{CF}_{4}$ gas [7]. Hamamatsu $\mathrm{Co}$. has already experience coupling the Bialkali photocathodes to these gases.

(a)

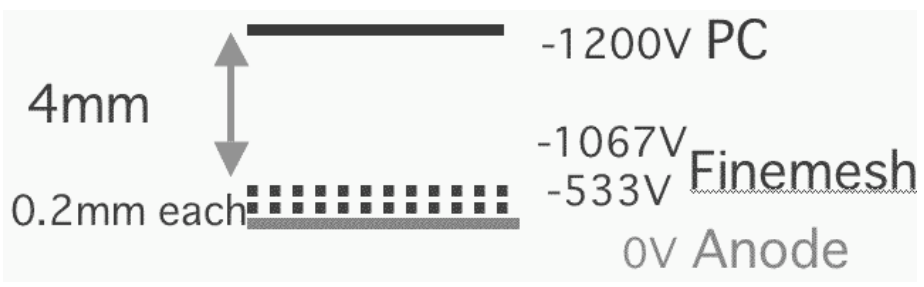

(b) 


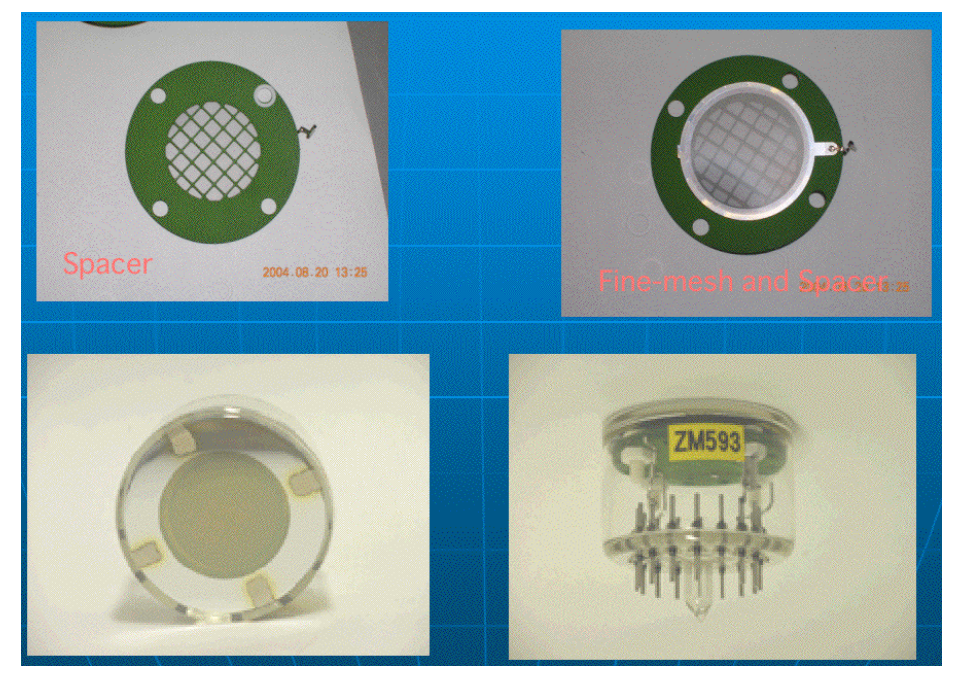

Fig. 11. (a) A double-Micromegas (mesh) prototype constructed by Hamamatsu operating with a Bialkali photocathode and $90 \% \mathrm{Ar}+10 \% \mathrm{CH}_{4}$ at 1 bar. (b) The picture shows details of the s.s. mesh support, a standard glass envelope with feedthrouhs.

The first results with the prototype of Fig. 11, performed by Hamamatsu and the Tokyo Metropolitan University is encouraging. For example, the photocathode performs well after it has been exposed to a P-10 gas, as can be see in Fig. 12a, which shows a measurement of the QE of Bialkali photocathode in: (i) First, $20.8 \%$ in vacuum, (ii) then $13 \%$ in the gas, and (iii) finally $20 \%$ in vacuum again. The QE returns to a nominal vacuum. Lower QE in the P-10 gas can be explained by a photoelectron backscattering in Argonbased gas (see above discussion).

The detector works well in both $\mathrm{P}-10$ and $90 \% \mathrm{Ar}+10 \% \mathrm{CF}_{4}$ gas. Two mesh solutions were tried $(25 \mu \mathrm{m} \&$ $34 \mu \mathrm{m})$. The detector reached a gain of $\sim 6 \times 10^{3}$ with a coarser pitch mesh, and $\sim 2 \times 10^{2}$ with a fine one. This is not yet good enough for a single photoelectron detection, however, it is a good progress. For example, Fig. $12 \mathrm{~b}$ shows a good result when the detector was connected to a $\mathrm{NaI}(\mathrm{Tl})$ crystal, and irradiated with a $\mathrm{Cs}^{137}$ source obtain the result shown above. Fig. 12c shows the first results of long-term photocathode aging when exposed to a P-10 gas: the photocathode stable for one week, and then, when operate done month later, it gives the same performance.

However, when measuring gain, we do see a faster rise than a simple exponential, which indicates that in this particular structure we do have the secondary effects. This means that the two-mesh solution would not be a good single photon detector, i.e., we need to suppress the ion backflow with a MCP with angled holes. This part is in progress. 

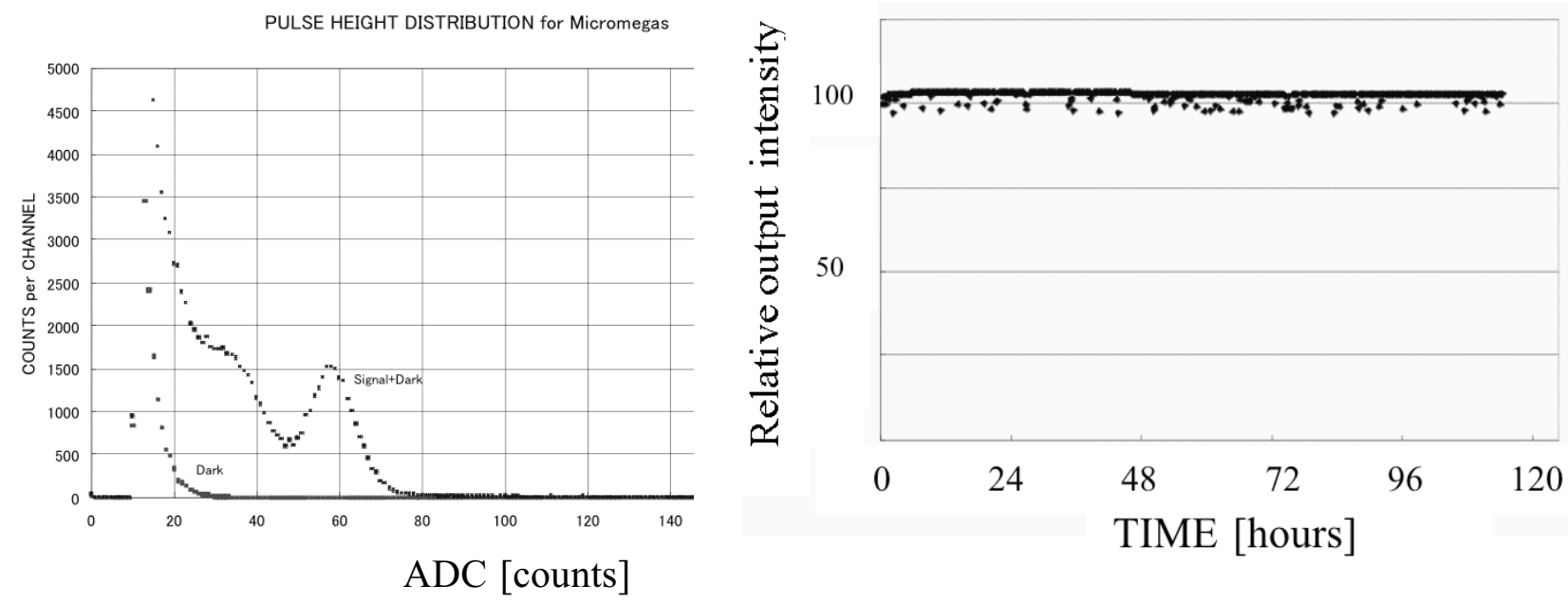

Fig.12. (a) Pulse height measurement with a $\mathrm{NaI}(\mathrm{Tl})$ crystal coupled to the double-mesh Micromegas photo-detector operating in the P-10 gas, and with a $\mathrm{Cs}^{137}$ source obtain the result shown above. (b) Photocathode aging when exposed to a P-10 gas: (i) the photocathode stable for one week; (ii) when operated one month later, it gives the same performance.

\section{DISCUSSION}

Table 1 attempts to compare pros and cons of two approaches to MCP-based detectors. There are many clear advantages of the vacuum-based MCP detectors such as the Burle multi-anode MCP-PMT. They are well established, at least in the R\&D efforts if not in a large experiment, and deliver a superior timing resolution approaching 50-70ps. However, there are still many questions left to answer. For example, the aging and reliability issues, the overall cost, or ability to retain a good timing resolution in a large system of $\sim 25 \mathrm{k}$ channels, which would be required for the good imaging system such as the Focusing DIRC. The gaseous detectors based on the MCP concept have many similar questions to answer, if not more. For example, a compatibility of the Bialkali photocathode with a gas, aging, timing resolution, etc.

Table 1 - Comparison of vacuum and gaseous MCP-based detectors

\begin{tabular}{|l|l|l|}
\hline Issue & Vacuum-based MCPs & Gaseous-based MCPs \\
\hline Operation with Bialkali & Well established & Needs very clean gas \\
\hline Main cause of aging & $\begin{array}{l}\text { Ion bombardment of } \\
\text { photocathode }\end{array}$ & $\begin{array}{l}\text { Secondary processes during ion } \\
\text { recombination }\end{array}$ \\
\hline Timing resolution $\left(1 \mathrm{pe}^{-}\right)$ & $\mathbf{\sigma} \sim \mathbf{3 0 - 6 0 p s ~ r e a c h e d ~}$ & Still to be measured \\
\hline Collection efficiency & Less than 70\% & Should be better than 98\% \\
\hline Magnetic field $(\mathrm{B}=15 \mathrm{kG})$ & Requires 6-10 $\boldsymbol{\mu m}$ dia. holes & $\mathbf{5 0} \boldsymbol{\mu m}$ dia. holes are OK \\
\hline Ion generating process & Residual gas bombardment & Avalanches in gas \\
\hline $\begin{array}{l}\text { Method to limit ion effects } \\
\text { at the photocathode }\end{array}$ & $\begin{array}{l}\text { Chevron arrangement of holes } \\
\left(6-12^{\circ} \text { inclination }\right)\end{array}$ & $\begin{array}{l}\text { We propose: Angled holes in a } \\
\text { direction of electron's Lorentz angle }\end{array}$ \\
\hline Cost & $\begin{array}{l}\text { Expensive if one chooses “non- } \\
\text { standard” parameters }\end{array}$ & Standard hole sizes \& angles \\
\hline
\end{tabular}


F. Sauli questioned if the inclined holes would slowly charge up causing the MCP electric field to align itself with the hole axis. Such effect would prevent a proper operation in the magnetic field. Although it is true that the resistivity of the present MCP material is high enough that it is causing a similar discharge time constant as that of the quadruple-GEM detector [see Fig. 8 in Ref. 1], as we said in the introduction, we do not plan to operate the MCP with higher gain than 10-20, while the rest of the required gain would be provided by the metallic Micromegas. We have performed a "magnetic field off" measurement aimed at searching for the long-term charging effects. In this test, the voltage across the MCP was set so that the gain would be $\sim 20$, if the field would slowly align itself with the hole axis. If there is a slow alignment of the electric field with the hole axis due to charging, the gain would grow in time. No increase in both the anode and the cathode currents were observed even after two weeks of continuous operation. We could not induce such effect even with a variable background intensity generated by the UV lamp being switched on/off. However, we have not done yet a similar "magnetic field on" tests, where one would see an opposite effect, i.e., the loss of electron transmission in time. Although we do not see any evidence of the long-term charging effect in our measurements, we do plan to replace the present material with a lower resistivity soda [5] glass in future.

\section{CONCLUSION}

This paper shows for the first time, to our knowledge, that it is possible to either eliminate the ion feedback entirely, or, reduce it to a very small level, simply by aligning the MCP holes with the electron Lorentz angle. The ions follow a different path and are caught on the MCP holes. In a geometry, where one can arrange the MCP electric field to have an angle with the magnetic field, one can indeed achieve this, and this paper provides a proof. For example, such geometry could be easily achieved in the Focusing DIRC by suitably rotating the focal plane.

Our initial tests with Bialkali photocathode are encouraging. The photocathode seems to coexist with a gas filling. More tests are needed to establish proof of principle though.

The ideas presented in this paper could be used in some other areas of detector physics, including, for example, limitation of the ion feedback in TPC. One could achieve a similar ion blockage for 4-6 Tesla, if one rotates the MCP by $10^{\circ}$ and increases thickness of the MCP.

\section{ACKNOWLEDGMENT}

We would like thank S. Biagi for useful comments how to run the Magboltz program, and M. McCulloch for help to construct the detector.

\section{REFERENCES}

[1] J. Va'vra and T. Sumiyoshi, "Single electron Amplification in a Single-MCP+Micromegas+Pads Detector," IEEE Trans. Nucl. Sci., vol. 51, No. 5, October 2004, and Nucl. Instr. \& Meth., A435(2004)334.

[2] Abe at al., Nucl. Instr.\&Meth., A343(1994)74, and A300(1991)501.

[3] J. Va'vra and A. Sharma, Nucl. Instr. \& Meth., A478(2002)235.

[4] C. Altunbas et al, Nucl. Instr. \& Meth., A490(2002)177, and A. Magnon et al., Nucl. Instr. \& Meth., A478(2002)210. 
[5] F. Tokanai et al., "Soda Glass Capillary Plate Gas Detector," IEEE Trans. Nucl. Sci., N25-8, October 2004, Rome, Italy.

[6] A. Breskin et al., Nucl. Instr.\&Meth., A483(2001)670.

[7] S. Biaggi, "Accurate Solution of the Boltzmann Transport Equation," Nucl. Instr. \& Meth., A273(1988)533-535. 